\title{
Erratum: Principles for Sensitive and Robust Biomolecular Interaction Analysis: The Limits of Detection and Resolution of Diffraction-Limited Focal Molography [Phys. Rev. Applied 11, 014056 (2019)]
}

\author{
Andreas Frutiger, ${ }^{1}$ Yves Blickenstorfer, ${ }^{1}$ Silvio Bischof, ${ }^{1}$ Csaba Forró, ${ }^{1}$ Volker Gatterdam, ${ }^{1}$ \\ János Vörös, ${ }^{1}$ Matthias Lauer, ${ }^{2}$ and Christof Fattinger ${ }^{2}$ \\ ${ }^{1}$ Laboratory of Biosensors and Bioelectronics, Institute of Biomedical Engineering, ETH Zrich, 8092 Zrich, \\ Switzerland \\ ${ }^{2}$ Roche Pharma Research and Early Development, Roche Innovation Center Basel, 4070 Basel, Switzerland
}

(Received 6 May 2021; published 30 June 2021)

DOI: 10.1103/PhysRevApplied.15.069901

After publication of the paper [1] we noted a few typing errors as well as a unit error in the stated numerical values of the sensitivity. The typing errors do not have any implications on the statements in the paper.

- Page 22 top right corner: "polarizibilty" is spelled wrong, it should read "polarizability".

- Figure 16: $f_{\text {eff }}$ is wrongly indicated. It should only go until the apparent surface. The corrected Figure 1 is stated in this document.

- Equation H1: The fraction was flipped. The equation should read: $d_{a}=\frac{n_{o}}{n_{\mathrm{s}}} d_{r}$

- Equation K2: The convolution sign and one of the integration variables is missing. The correct equation reads:

$$
\frac{I_{\text {sig }}}{I_{\text {Airy,max }}}=\left.\left(\left(\frac{2 J_{1}(r)}{r}\right)^{2} * \frac{\left(\frac{2 J_{1}(r)}{r}\right)^{2}}{\int_{0}^{2 \pi} \int_{0}^{\infty}\left(\frac{2 J_{1}\left(r^{\prime}\right)}{r^{\prime}}\right)^{2} r^{\prime} d r^{\prime} d \varphi}\right)\right|_{r=0}
$$

(a)

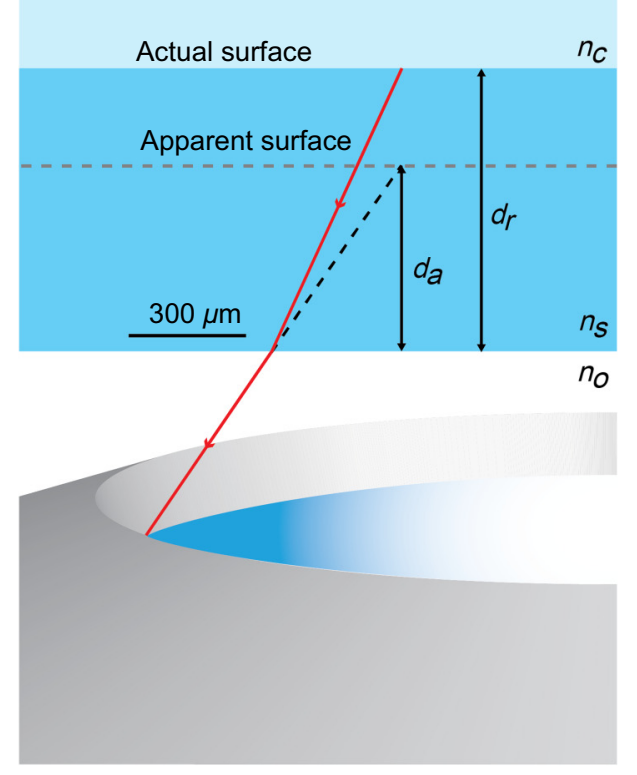

(b)

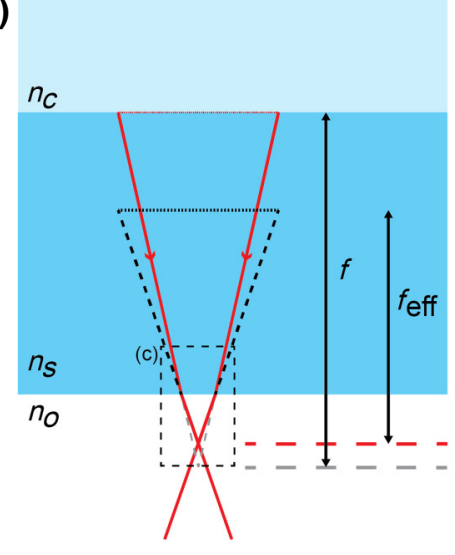

(c)

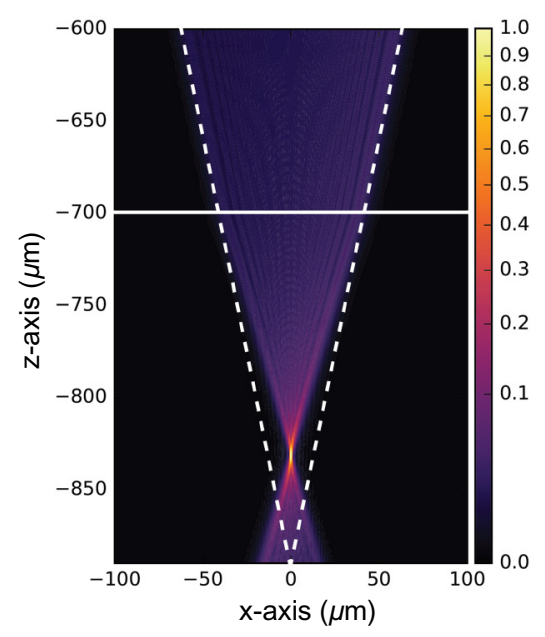

FIG. 1. Corrected Figure 16b. In the original figure the effective focal distance $f_{\text {eff }}$ was drawn all the way up to the surface. This is not true. The effective focal distance is shorter as the light rays from the surface are deflected according to Snell's law and the virtual image of the surface is formed within the glass as indicated here. The change has no implications on the statements in the paper. 
- Equation E5: The variable $A$ is not mentioned in the text. It is the amplitude of the electromagnetic field obtained from the ansatz in chapter 1.3 of Marcuse, D. Theory of dielectric optical waveguides. (Academic Press, 1974). [2] (Variable $\mathrm{Z}_{w}$ is the impedance of vacuum)

- Page 41 In the sentence: "Inserting the values for gold at $700 \mathrm{~nm}$ and using the calculated effective index of 1.41, one obtains $\mathrm{S}_{\Gamma}=1.16 \cdot 10^{5}\left(\mathrm{~mm}^{2} / \mathrm{pg}\right)$." The units of the sensitivity were wrong. The value stated was in $\mathrm{m}^{2} / \mathrm{kg}$. The sensitivity in $\left(\mathrm{mm}^{2} / \mathrm{pg}\right)$ is $\mathrm{S}_{\Gamma}=1.16 \cdot 10^{-4}$. The same is also true for the sensitivity of focal molography on page 42 , which should read $\mathrm{S}_{\Gamma}=1.1\left(\mathrm{~mm}^{2} / \mathrm{pg}\right)$. The unit error does not affect any figures or statements in the paper.

[1] A. Frutiger, Y. Blickenstorfer, S. Bischof, C. Forró, M. Lauer, V. Gatterdam, C. Fattinger, and J. Vörös, Principles for Sensitive and Robust Biomolecular Interaction Analysis: The Limits of Detection and Resolution of Diffraction-Limited Focal Molography, Phys. Rev. Applied 11, 014056 (2019).

[2] D. Marcuse, Theory of dielectric optical waveguides, Quantum electronics - principles and applications (Academic Press, 1974). 